\title{
Addressing Intersecting Housing and Overdose Crises in Vancouver, Canada: Opportunities and Challenges from a Tenant-Led Overdose Response Intervention in Single Room Occupancy Hotels
}

\author{
Geoff Bardwell • Taylor Fleming • \\ Alexandra B. Collins • Jade Boyd • Ryan McNeil \\ Published online: 2 August 2018 \\ (C) The New York Academy of Medicine 2018
}

\begin{abstract}
We examined the acceptability, feasibility, and implementation of the Tenant Overdose Response Organizers program (TORO) - a tenant-led naloxone training and distribution intervention. This pilot project was implemented in privately owned single room occupancy (SRO) hotels that were disproportionately affected by overdose in Vancouver's Downtown Eastside (DTES) neighborhood. Semi-structured qualitative interviews were conducted with 20 tenants who had participated in a TORO training session and administered naloxone to someone in their SRO hotel or had overdosed in their SRO hotel and received naloxone from another tenant. Focus groups were conducted with 15 peer workers who led the TORO program in their SRO building. Interviews and focus groups were transcribed and analyzed thematically. Ethnographic observation at SRO hotels involved in the intervention was also co-led with peer research
\end{abstract}

G. Bardwell · T. Fleming - A. B. Collins · J. Boyd •

R. McNeil

British Columbia Centre on Substance Use, St. Paul's Hospital,

608-1081 Burrard Street, Vancouver, British Columbia V6Z 1Y6, Canada

G. Bardwell $\cdot$ J. Boyd $\cdot$ R. McNeil $(\bowtie)$

Department of Medicine, St. Paul's Hospital, University of British Columbia, 608-1081 Burrard Street, Vancouver, British Columbia V6Z 1Y6, Canada

e-mail: rmcneil@cfenet.ubc.ca

A. B. Collins

Faculty of Health Sciences, Simon Fraser University, 8888

University Drive, Burnaby, British Columbia V5A 1S6, Canada assistants. Ten SROs were included in the study. The level of acceptability of the TORO program was high, with participants describing the urgency for an intervention amid the frequency of overdoses in their buildings. Overdose response training enhanced participants' knowledge and skills, and provided them a sense of recognition. Additionally, the TORO program was feasible in some buildings more than others. While it provided important training and engaged isolated tenants, there were structural barriers to program feasibility. The implementation of the TORO program was met with some successes in terms of its reach and community development, but participants also discussed a lack of emotional support due to overdose frequency, leading to burnout and vulnerability. Our findings suggest that the TORO program was affected by social, structural, and physical environmental constraints that impacted program feasibility and implementation. Despite these constraints, peer-led in-reach overdose response interventions are effective tools in addressing overdose risk in SROs. Future housing interventions should consider the intersecting pathways of overdose risk, including how these interventions may exacerbate other harms for people who use drugs. Further research should explore the impacts of environmental factors on overdose response interventions in other housing contexts.

Keywords Housing · Single room occupancy hotels · Risk environments · Overdose response $\cdot$ Safer environment interventions $\cdot$ Peer naloxone programs 


\section{Introduction}

North America is currently experiencing an overdose epidemic [1-3]. In 2016, there were over 42,000 opioid overdose deaths in the USA [4]. Recently, there has been an upward trend in the number of overdoses driven by illicit fentanyl [5-9]. Previous research has demonstrated associations between overdose and housing vulnerability including: people who use drugs (PWUD) experiencing homelessness [10], those residing in single room occupancy (SRO) hotels [11] or neighborhoods with income inequalities [12], and those using drugs indoors [13]. These studies call for targeted interventions, including overdose response training for PWUD.

Multiple studies have examined how environmental factors create risks for PWUD and the need for safer environment interventions (SEI) to reduce these risks [14-21]. There remains a gap in the literature on the potential for peer-led overdose response interventions targeting high-risk housing environments and their potential role in responding to the overdose crisis [22]. Teaching overdose responses to PWUD is an effective risk reduction strategy that builds on their existing knowledge [23]. Naloxone programs targeting PWUD have additional benefits including improved self-esteem, empowerment, and strengthened relationships [24-28]. We build on this research by focusing on the ways in which contextual factors impact the experiences of PWUD and SEI implementation in SROs.

Drawing on Rhodes' risk environment framework, we examine environmental factors shaping overdoserelated risks in SROs. Risk is produced through three environment typologies: physical (e.g., building conditions), social (e.g., stigma), and structural environments (e.g., housing policies) [29]. Environmental changes can reduce overdose risks through the implementation of SEIs (e.g., harm reduction interventions) [30], and housing environments represent a key implementation setting given their links to overdose.

British Columbia (BC), Canada, is experiencing an overdose epidemic [31]. Despite the scale-up of overdose-focused interventions, BC experienced over 1400 overdose-related deaths in 2017, with the majority (94\%) occurring in housing environments (e.g., emergency shelters, private housing, social housing) [32], including SROs, which provide housing to marginalized communities. Vancouver, BC, is undergoing overlapping overdose and housing crises, with an over $30 \%$ increase in homelessness since 2014 [33], driven by socio-structural determinants, including gentrification [34, 35], insufficient social assistance rates [36, 37], and housing policy shortcomings [38]. SROs remain one of the only accessible housing options for vulnerable PWUD - that is, buildings providing single room units to tenants along with shared bathrooms, some lacking kitchens. SROs are largely concentrated in the Downtown Eastside (DTES) and have considerable variation in their physical conditions, which impacts the well-being of PWUD [16, 17, 39, 40].

In response to a significant number of overdoses in privately owned SROs in the DTES, the DTES SRO Collaborative [41], which aims to improve SRO conditions and tenants' rights, received funding to implement the Tenant Overdose Response Organizers (TORO) program. This pilot project employed a peer-led approach supported by a public health nurse to provide in-reach (i.e., health services offered inside buildings) naloxone training and distribution to tenants in 12 private SROs with the highest rates of overdose, as identified by overdose surveillance data. One to two tenants from each building were hired for $5 \mathrm{~h}$ per week as "TOROs" (hereafter cited as "tenant organizers"), and were the main overdose response contacts in their buildings. Along with a public health nurse and DTES SRO Collaborative staff, tenant organizers recruited tenants to participate in onsite naloxone training, which included group education on opioid overdoses, overdose response training, and naloxone kits. Tenants unable to attend the group training could request one-on-one training with the tenant organizers. Because three buildings were unable to complete the intervention due to building-wide evictions associated with renovations, one additional building was added to the intervention, for a total of ten SROs. Building management were approached by the DTES SRO Collaborative coordinator and the public health authority prior to intervention implementation.

We sought to examine the acceptability, feasibility, and implementation of the TORO program, as well as its impact on responses to overdoses in privately owned SROs. We also examine how SROs produce tenant vulnerability and overdose risk environments vis-à-vis physical, social, and structural factors.

\section{Methods}

This qualitative study was undertaken between June and September 2017 and involved semi-structured 
interviews, focus groups, and ethnographic observation. Interviews captured SRO tenants' experiences of overdose and the intervention; focus groups with the tenant organizers sought to understand intervention implementation and limitations; and ethnographic observation focused on building conditions, naloxone trainings, and social interactions. Ethical approval was obtained through the University of British Columbia/Providence Health Care Research Ethics Board.

For interviews, we aimed to recruit three tenants from each SRO $(n=30)$ and developed a recruitment checklist to ensure demographic diversity. Socio-structural constraints (e.g., evictions, targeting of PWUD) created recruitment challenges, and thus only 20 tenants from eight SROs were recruited; however, we were still able to reach a diverse sample (see Table 1 for participant demographics). Participants were recruited by word-ofmouth and referred to our research team by the tenant organizers. Participants were required to have: (a) completed the TORO training and administered naloxone in their building ( $n=10)$, or (b) overdosed in a TORO building and were administered naloxone by a tenant $(n=10)$. These criteria were used to evaluate program effectiveness, including how overdose situations were handled and experienced. Two focus groups were conducted with tenant organizers $(n=15)$ to understand their work experiences. All participants provided informed consent and received \$30 (CAD) honoraria. A guide was used to facilitate the interviews, which were audio-recorded. Interviews were transcribed verbatim and checked for accuracy.

Additionally, along with peer research assistants (i.e., hired affected community members), the lead author completed $50 \mathrm{~h}$ of ethnographic observation in the buildings to explore the environmental conditions that may impact overdose risk and to enhance validity [42].

Table 1 Interview participant demographics $(n=20)$

\begin{tabular}{llr}
\hline Gender & Men & 11 \\
Ethnicity & Women & 9 \\
& White & 13 \\
& Indigenous/racialized & 6 \\
Sexuality & Refused & 1 \\
& Straight & 18 \\
Administered naloxone & Sexual minority & 2 \\
Overdosed and received naloxone & & 10 \\
\hline
\end{tabular}

Access to SROs was obtained through conversations with building staff and invitations by the tenant organizers. The lead author took field notes during observations to contextualize the research.

We utilized a team approach in developing the data coding framework. Each author reviewed a portion of the transcripts individually and then we met as a group and developed a coding framework using a priori themes and emerging thematic categories [43]. NVivo 11 was used to code the data into themes and subthemes for analysis. Each author coded four transcripts, and coding was reviewed and verified by the lead author to enhance reliability and trustworthiness. We also met to discuss and revise the coding framework, which served as a reliability check. The risk environment framework was used to frame emerging themes during analysis. Findings were presented to the DTES SRO Collaborative to solicit feedback and strengthen interpretive validity.

\section{Results}

Acceptability of TORO

\section{Urgency and Receptiveness}

Prior to the TORO implementation, participants described overdose response within their buildings as "chaotic" due to frequent overdoses. The limited capacity of tenants to respond effectively to overdoses, alongside the uneven availability of naloxone, posed challenges. Multiple participants discussed the difficulty in identifying someone who had a naloxone kit, training, and the confidence to respond prior to implementation. One tenant organizer noted:

I responded to six overdoses prior to the program starting, and when I was responding then it was like 'holy shit, who's got Narcan [i.e., naloxone]?' I'd send my girlfriend running around to find somebody who had Narcan so I could administer it. (Participant \# 6, focus group \# 2)

Given the severity of the overdose crisis, participants reported receptiveness to the TORO program as a timely and important mechanism for tenant overdose response. One participant explained: 
I think a lot of people are really aware and it's nice when you see everyone's yelling like 'Narcan! Narcan!' and people are very quick to jump up and help and then there's like ten people showing up with Narcan kits. (Participant \# 1)

Participants attributed increased awareness and capabilities to deal with overdoses in their buildings to the TORO program.

\section{Enhanced Knowledge, Skills, and Recognition}

The program enabled tenants to develop a variety of skills, including overdose response, communication, and interpersonal skills. Group trainings were an effective way for participants to collectively learn about issues related to opioid overdose, and for the tenant organizers and nurse to dispel any overdose myths. Some participants discussed feeling a sense of empowerment through having the proper life-saving skills. One participant explained:

The knowledge is quite good. If you go into a building that has the TORO project, everyone's calm and relaxed. But if you go into a building where someone's OD'd and there's not any knowledge, then it's just absolute chaos. (Participant \# 4, focus group \# 1)

Participants further described the TORO program as a way to come together to respond to the overdose crisis, and expressed a sense of acknowledgement from community members, including neighbors and first responders, due to the program. For example:

I had ambulance attendants that came to help pick up the person that OD'd in the hall that I had to help with, and he said, 'How did you know what to do?' I said, 'Well, I just learned it at the TORO program.' And he goes, 'I've heard about that. It's great having you guys stepping up and getting out into the community and helping.' He said it's cut their job down a lot, because of knowing what this program has taught a lot of people, and it's saving lives. (Participant \# 3, focus group \# 2)

Increased opioid overdose knowledge and skills from the program qualified tenants to respond more effectively to overdoses and contribute to wider community responses.

\section{Feasibility of TORO}

\section{Engaging Isolated Tenants}

Our findings demonstrate the feasibility of the TORO program to engage socially isolated PWUD for the purposes of naloxone training and distribution. Among participants, the program was perceived as life-saving in the midst of an ongoing crisis, and the opportunity to access naloxone training and kits within their SROs was positioned as protective against fatal overdose. Although many participants reported social isolation, the implementation of naloxone programming into these SROs extended this overdose response strategy to PWUD who were not attached to other services or those who previously were not concerned about overdosing or requiring training. For example:

For a long time I was the guy that didn't bother. I think I wanted a [naloxone] kit for myself, really. Because now I have a kit in my room and, if something happens to me, at least there's something in my room, right? I don't have to rely on somebody else having one. (Participant \# 6)

In some cases, participants reported challenges in attracting tenants, but noted that group and individual training sessions demonstrated intervention feasibility for hard-to-reach tenants.

\section{Environmental Barriers to Effective Program Implementation}

Physical, social, and structural conditions undermined the housing security of PWUD, including tenant organizers. As noted during ethnographic observation, physical conditions in the participating SROs were dire, with broken windows and doors that impacted the safety of residents and a lack of access to water for drinking or bathing due to plumbing issues (Figs. 1 and 2). These SROs also lacked harm reduction rooms and supplies.

Housing security was also jeopardized by social and structural conditions, such as a lack of acceptance and support from some building management and landlords. Evictions from private SROs were commonplace due to the prejudicial targeting of PWUD and absence of legal 


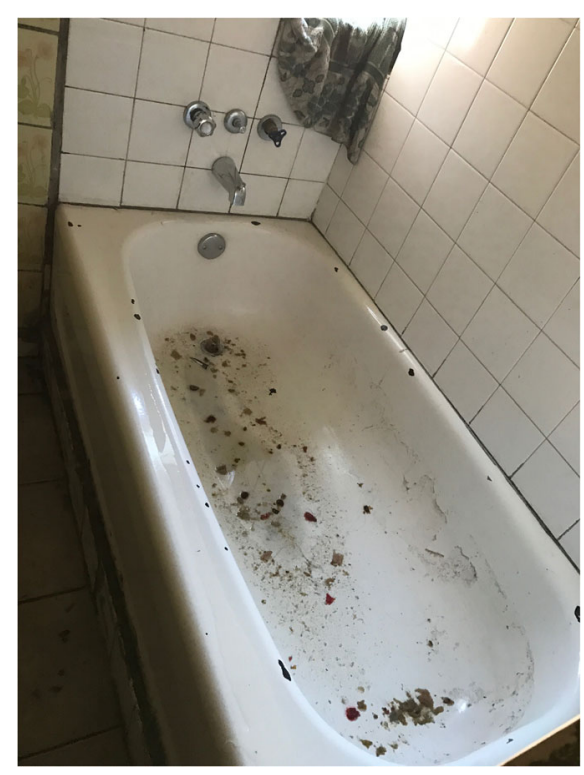

Fig. 1 Plugged bathtub at SRO \#1

knowledge or access to legal mechanisms to protect tenancy rights. While many participants reported routine harassment and threats of eviction from managers and landlords, they emphasized that participating in naloxone training and administrating naloxone during an overdose could further undermine their housing security by associating tenants with drug use. Here, managers and landlords exploited the vulnerability of PWUD to evict large numbers of tenants. One participant explained how, although he had regular eviction threats, his involvement with the TORO project formally identified him as a PWUD and thereby exacerbated his housing vulnerability: "Well the staff were getting upset with me and they said, 'the next time you Narcan somebody we're gonna evict you.' It

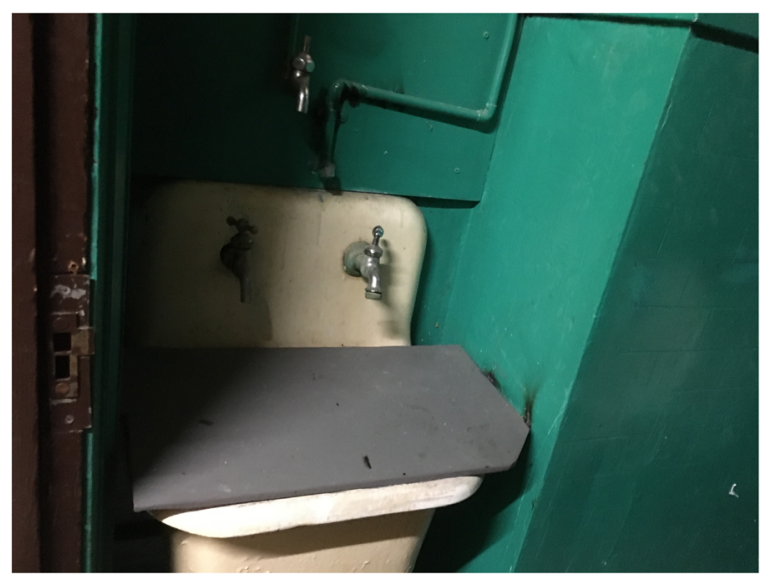

Fig. 2 Covered broken sink at SRO \#8 was an idle threat because they evicted me about once or twice a week officially, it was just ridiculous" (Participant \# 13). We observed some staff and managers questioning the TORO program, with one staff telling the lead author to "get the fuck out of the building" for talking with tenants. Some managers and landlords were viewed as more supportive of the intervention due to the recognition of the severity of the overdose crisis. This support was also evident during ethnographic observation, where, for example, one building manager spoke about how he attended the training and proactively talks to tenants about naloxone. Here, tenant organizers positioned such acceptance as necessary to foster social-structural conditions that enabled the effective implementation of the program, and minimized risks for participants. For example:

They have a new manager now. They fired the old manager and the new manager attended the last Narcan training. He's a really nice guy and he wants to be involved. He's pretty open to the training. (Participant \# 19)

\section{Implementation Opportunities and Challenges}

\section{Community Development}

The TORO project supported the development of mutual support among tenants. Throughout the overdose crisis, naloxone has become a symbol of shared experience, with participants discussing the responsibility they feel for themselves and others. Participants emphasized the importance of a peer-run intervention, expressing comfort in having a peer respond to overdoses in comparison to emergency responders. One participant explained that a neighbor responding to overdose is less stigmatizing and preferable because "it's like your neighbor... [a] part of the community" (Participant \# 19).

The opportunity to respond effectively to overdoses was affirming and allowed participants to demonstrate leadership. Tenant organizers expressed a sense of accomplishment in bringing the community together by training tenants, their peers, and, in some cases, building managers and staff to respond to overdoses:

Everybody's got everybody's back out there. Always. When a guy goes down, we're hustling hard. And everybody [is] loving that person, you know? They show him love, man, and they're 
loving him to come back, right? Every time.

Those people have hearts. (Participant \# 18)

Participants articulated a sense of pride in coming together within the context of an overdose crisis.

\section{Lack of Emotional Support}

Almost all tenant organizers highlighted how their roles created increased emotional distress. For example, participants emphasized that they were emotionally taxed by the high stakes associated with responding to overdoses. Tenant organizers worked extended hours without additional pay to ensure they were always available. Participants perceived themselves as needing to remain "on call" and indicated that they were "getting burned out." Such feelings were exacerbated by the severity of the overdose crisis, as participants regularly addressed the overdoses of friends. One participant described:

It gets to you after a while, it really does. The the last person that I did [i.e., administered naloxone] I could not bring him back and he was my buddy. By the time the ambulance came, he already stopped breathing...I've known him over 30 years. (Participant \# 20)

The majority of participants highlighted the need for counseling supports to better manage ongoing emotional distress. Further, participants' accounts underscored how their vulnerability created challenges in the day-today implementation of the program (e.g., lack of access to phones to call emergency services).

\section{Discussion}

Findings suggest that the TORO program was an effective overdose response intervention in privately owned SROs despite environmental constraints. First, the sense of urgency stemming from overdose vulnerability led to a high degree of acceptability for SEIs among tenants. Second, while the TORO program was effective in engaging isolated populations, social-structural constraints impacted feasibility, including a lack of acceptance and support from building management and owners in multiple SROs. Third, while program implementation was effective for community development, a lack of emotional support led to burnout.
The severity of the housing crisis in Vancouver means that PWUD often live in dire social, structural, and physical environments within SROs that compromise their health $[16,17,39,40,44]$. That these environments produced overdose vulnerability and framed housing-based overdose responses is important to consider in the context of the overdose crisis. Past studies have explored correlations between overdose risk and built environments [45], impoverished neighborhoods [46, 47], and a lack of adequate housing [48]. Our research builds on these studies by characterizing private SROs as overdose risk environments in which several factors intersect to produce overdoses, including: observed building conditions unfit for housing and unsupportive of harm reduction (physical); routine stigmatization and harassment and the emotional burden of living in high overdose environments (social); and drug criminalization occurring alongside unsupportive building management (structural). Taken together, these environmental factors function to not only shape overdose risks of PWUD, but pose challenges to the implementation of overdose-focused interventions and underscore the need for targeted SEIs in SROs [11].

Past research on peer naloxone programs illustrates a variety of benefits to these programs beyond overdose responses, including responsibility, empowerment, and improved self-esteem [24, 26-28]. Our study expands on this by not only highlighting the benefits and effectiveness of this novel peer-led SEI, but also problematizing the intervention given the adverse effects of the physical, social, and structural environments. While the TORO project demonstrated effectiveness in addressing overdose in high-risk environments, this was sometimes at the expense of the tenant organizers leading the intervention who both worked and lived in these risk environments, where they were exposed to frequent overdoses. SROs where building staff and management either participated in naloxone training or supported the intervention in their buildings were thus different than buildings where tenants experienced routine harassment, targeting, and eviction threats. In the latter case, tenantled interventions are constrained by these factors which affected tenant organizers' ability to provide naloxone training or administer naloxone without negative consequences.

Against this backdrop, it is important to consider that, while SEIs may reduce risk in particular ways, they may also reconfigure environments in ways that increase vulnerabilities. This was evident in that the intervention 
built capacity to engage isolated tenants and respond to overdoses, while simultaneously identifying specific tenants as PWUD given their participation in the TORO project and thereby exacerbated housing vulnerabilities. This study therefore adds a critical dimension to understanding the role of SEIs in mitigating impacts of contextual forces. During SEI implementation in risk environments, explicit attention needs to be paid to the multiple environmental constraints under which these interventions operate. Past qualitative feasibility studies on harm reduction interventions (e.g., supervised injection) have found that support from other stakeholders (e.g., building owners) is key for optimal service delivery [49]. While SEIs play an integral role in reshaping risk environments, they do not exist in a vacuum and must be accompanied by other efforts to address factors constraining their effectiveness (e.g., laws, social stigma). For example, in SROs, there is an urgent need to link intervention directly to advocacy to address environmental constraints and thereby optimize overdose responses. Even when deployed to recreate more positive environmental conditions, SEIs alone will be insufficient in addressing underlying physical, social, and structural conditions and will require recognition and action to address factors that create risk environments at the macro-level.

There were some limitations to our study. First, while we made an effort to reach a diversity of tenants, participant experiences may not reflect those of other SRO tenants. Second, this study examined ten SROs in the DTES and the characterization of these buildings may not be applicable to others, including those governed by non-profit organizations. Third, bylaws, laws, and policies that impact SROs in our study are Vancouverspecific and therefore may not be applicable to other jurisdictions.

In conclusion, our study highlights the need for the implementation of SEIs within SROs to address the risks associated with overdose and housing vulnerabilities. Despite the social, structural, and physical environmental constraints that impacted the TORO project, peer-led in-reach overdose response interventions are effective tools in addressing overdose in SROs. Future SEIs in housing environments would benefit from greater consideration of the multiple intersecting risk environments and how SEIs may unintentionally exacerbate other risks. Coinciding with future housing SEIs, additional research is needed to explore the impacts of environmental factors on the acceptability, feasibility, and implementation of peer-led interventions in other housing environments such as non-profit SROs, transitional housing, and emergency shelters.

Acknowledgements We would like to thank study participants for their contributions to this research. We also thank current and past staff and peer research assistants at the British Columbia Centre on Substance Use for their research and administrative assistance. Thank you to the DTES SRO Collaborative and Vancouver Coastal Health for allowing us to conduct this research.

This study was supported by funding from the City of Vancouver and the US National Institutes of Health (R01DA044181). Geoff Bardwell is supported by a Mitacs Elevate Postdoctoral Fellowship from Mitacs Canada. Alexandra Collins is supported by a Mitacs Award through the Mitacs Accelerate Program. Ryan McNeil is supported by awards from the Michael Smith Foundation for Health Research and the Canadian Institutes of Health Research.

\section{References}

1. Government of Canada. Apparent opioid-related deaths. 2017 ; https://www.canada.ca/en/healthcanada/services/substance-abuse/prescription-drugabuse/opioids/apparent-opioid-related-deaths.html . Accessed November 20, 2017.

2. Rudd RA, Aleshire N, Zibbell JE, Gladden MR. Increases in Drug and Opioid Overdose Deaths—United States, 2000 2014. MMWR Morb Mortal Wkly Rep. 2016;64(50):137882.

3. King NB, Fraser V, Boikos C, Richardson R, Harper S. Determinants of increased opioid-related mortality in the United States and Canada, 1990-2013: a systematic review. Am J Public Health. 2014;104(8):e32-42.

4. Centers for Disease Control and Prevention Data Brief 294. Drug overdose deaths in the United States, 1999-2016. The United States: National Center for Health Statistics. Hyattsville, MD 2017.

5. Gladden RM, Martinez P, Seth P. Fentanyl law enforcement submissions and increases in synthetic opioid-involved overdose deaths -27 States, 2013-2014. MMWR Morb Mortal Wkly Rep. 2016;65(33):837-43.

6. Compton W. Research on the use and misuse of fentanyl and other synthetic opioids. 2017; https:/www.drugabuse. gov/about-nida/legislative-activities/testimony-tocongress $/ 2017 /$ research-use-misuse-fentanyl-othersynthetic-opioids. Accessed November 7, 2017.

7. Bode AD, Singh M, Andrews J, Kapur GB, Baez AA. Fentanyl laced heroin and its contribution to a spike in heroin overdose in Miami-Dade County. Am J Emerg Med. 2017;35(9):1364-5.

8. O'Donnell JK, Halpin J, Mattson CL, Goldberger BA, Gladden RM. Deaths Involving Fentanyl, Fentanyl Analogs, and U-47700-10 States, July-December 2016. Morbidity and Mortality Weekly Report. 2017 Nov;66(43): 1197-202. 
9. National Drug Early Warning System Coordinating Center. The increase in fentanyl overdoses. United States: National Drug Early Warning System Coordinating Center;2016.

10. Seal KH, Kral AH, Gee L, Moore LD, Bluthenthal RN, Lorvick J, et al. Predictors and prevention of nonfatal overdose among street-recruited injection heroin users in the San Francisco Bay Area, 1998-1999. Am J Public Health. 2001;91(11):1842-6.

11. Davidson PJ, McLean RL, Kral AH, Gleghorn AA, Edlin BR, Moss AR. Fatal heroin-related overdose in San Francisco, 1997-2000: a case for targeted intervention. $J$ Urban Health : Bull N Y Acad Med. 2003;80(2):261-73.

12. Galea S, Ahern J, Vlahov D, et al. Income distribution and risk of fatal drug overdose in New York City neighborhoods. Drug Alcohol Depend. 2003;70(2):139-48.

13. Siegler A, Tuazon E, Bradley O'Brien D, Paone D. Unintentional opioid overdose deaths in New York City, 2005-2010: a place-based approach to reduce risk. Int $J$ Drug Policy. 2014;25(3):569-574.

14. Galea S, Ahern J, Vlahov D. Contextual determinants of drug use risk behavior: a theoretic framework. J Urban Health : Bull N Y Acad Med. 2003;80(4 Suppl 3):iii50-58.

15. Kerr T, Small W, Moore D, Wood E. A micro-environmental intervention to reduce the harms associated with drug-related overdose: evidence from the evaluation of Vancouver's safer injection facility. Int J Drug Policy. 2007;18(1):37-45.

16. Knight KR, Lopez AM, Comfort M, Shumway M, Cohen J, Riley ED. Single room occupancy (SRO) hotels as mental health risk environments among impoverished women: the intersection of policy, drug use, trauma, and urban space. Int J Drug Policy. 2014;25(3):556-61.

17. Lazarus L, Chettiar J, Deering K, Nabess R, Shannon K. Risky health environments: women sex workers' struggles to find safe, secure and non-exploitative housing in Canada's poorest postal code. Soc Sci Med(1982). 2011;73(11):1600-7.

18. McNeil R, Small W, Wood E, Kerr T. Hospitals as a 'risk environment': an ethno-epidemiological study of voluntary and involuntary discharge from hospital against medical advice among people who inject drugs. Soc Sci $\operatorname{Med}(1982)$. 2014;105:59-66.

19. Pauly B, Reist D, Belle-Isle L, Schactman C. Housing and harm reduction: what is the role of harm reduction in addressing homelessness? Int J Drug Policy. 2013;24(4):284-90.

20. Rhodes T, Watts L, Davies S, et al. Risk, shame and the public injector: a qualitative study of drug injecting in South Wales. Soc Sci Med(1982). 2007;65(3):572-85.

21. Small W, Rhodes T, Wood E, Kerr T. Public injection settings in Vancouver: physical environment, social context and risk. Int J Drug Policy. 2007;18(1):27-36.

22. Bardwell G, Collins AB, McNeil R, Boyd J. Housing and overdose: an opportunity for the scale-up of overdose prevention interventions? Harm Reduction J. 2017;14(1):77.

23. Sherman SG, Gann DS, Scott G, Carlberg S, Bigg D, Heimer R. A qualitative study of overdose responses among Chicago IDUs. Harm Reduction J. 2008;5:2.

24. Banjo O, Tzemis D, Al-Qutub D, Amlani A, Kesselring S. Buxton JA. A quantitative and qualitative evaluation of the British Columbia take home naloxone program. CMAJ Open. 2014;2
25. Bartlett N, Xin D, Zhang H, Huang B. A qualitative evaluation of a peer-implemented overdose response pilot project in Gejiu, China. Int J Drug Policy. 2011;22(4):301-5.

26. Marshall C, Perreault M, Archambault L, Milton D. Experiences of peer-trainers in a take-home naloxone program: Results from a qualitative study. Int J Drug Policy. 2017;41:19-28.

27. Mitchell K, Durante SE, Pellatt K, Richardson CG, Mathias $\mathrm{S}$, Buxton JA. Naloxone and the Inner City Youth Experience (NICYE): a community-based participatory research study examining young people's perceptions of the BC take home naloxone program. Harm Reduction J. 2017; 14:34.

28. Wagner KD, Davidson PJ, Iverson E, et al. "I felt like a superhero": the experience of responding to drug overdose among individuals trained in overdose prevention. Int $J$ Drug Policy. 2014;25(1):157-65.

29. Rhodes T. Risk environments and drug harms: a social science for harm reduction approach. Int J Drug Policy. 2009;20(3):193-201.

30. McNeil R, Small W. 'Safer environment interventions': a qualitative synthesis of the experiences and perceptions of people who inject drugs. Soc Sci Med(1982). 2014;106:151-8.

31. BC Coroners Service. Fentanyl detected in $81 \%$ of illicit drug deaths in B.C. in 2017. 2017; https://archive.news.gov. bc.ca/releases/news_releases_2017-2021/2017PSSG0049001545.htm. Accessed November 7, 2017.

32. British Columbia Coroners Service. Illicit drug overdose deaths in BC, January 1, 2007 - December 31, 2017. Br Columbia 31, College Park, MD 2018.

33. BC Non-Profit Housing Association \& M. Thomson Consulting. 2017 Homeless Count in Metro Vancouver. Burnaby, BC: Metro Vancouver Homelessness Partnering Strategy Community Entity;2017.

34. Ciccarone D, Bourgois P. Injecting drugs in tight spaces: HIV, cocaine and collinearity in the Downtown Eastside, Vancouver, BC Int J Drug Policy 2016;33:36-43.

35. Burnett K. Commodifying poverty: gentrification and consumption in Vancouver's Downtown Eastside. Urban Geography. 2014;35(2):157-76.

36. Tweddle A, Battle K, Torjman S. Welfare in Canada, 2015. Ottawa, ON: the Caledon institute of Social Policy;2016.

37. Klein S, Ivanova I, Leyland A. Long overdue: why BC needs a poverty reductin plan. Vancouver, BC: Canadian Centre for Policy Alternatives;2017.

38. Lee M. Getting serious about affordable housing: towards a plan for metro Vancouver. Vancouver, BC: Canadian Centre for Policy Alternatives;2016.

39. Evans L, Strathdee SA. A roof is not enough: unstable housing, vulnerability to HIV infection and the plight of the SRO. Int J Drug Policy. 2005;17(2):115-117.

40. Shannon K, Ishida T, Lai C, Tyndall MW. The impact of unregulated single room occupancy hotels on the health status of illicit drug users in Vancouver. Int J Drug Policy. 2006;17(2):107-14.

41. DTES SRO Collaborative Society. Tenant Overdose Response Organizers Program. 2017; https://dtescollaborative.org/toroproject/. Accessed September 8, 2017.

42. Creswell JW, Miller DL. Determining validity in qualitative inquiry. Theory Pract. 2000;39(3):124-30. 
43. Corbin J, Strauss A. Basics of qualitative research: techniques and procedures for developing grounded theory. Thousands Oaks, CA: Sage Publications; 2015.

44. Harbottle T. Cracking down on grim SRO Hotels 2011; https://thetyee.ca/News/2011/06/30/GrimSROs/. Accessed October 30, 2017.

45. Hembree C, Galea S, Ahern J, et al. The urban built environment and overdose mortality in New York City neighborhoods. Health Place. 2005;11(2):147-56.

46. Visconti AJ, Santos GM, Lemos NP, Burke C, Coffin PO. Opioid overdose deaths in the city and county of San Francisco: prevalence, distribution, and disparities. $J$ Urban Health : Bull N Y Acad Med. 2015;92(4):758-72.
47. Rowe C, Santos GM, Vittinghoff E, Wheeler E, Davidson P, Neighborhood-Level CPO. Spatial characteristics associated with lay naloxone reversal events and opioid overdose deaths. J Urban Health : Bull N Y Acad Med. 2016;93(1): 117-30.

48. Jenkins LM, Banta-Green CJ, Maynard C, et al. Risk factors for nonfatal overdose at Seattle-area syringe exchanges. $J$ Urban Health : Bull N Y Acad Med. 2011;88(1):118-28.

49. Bardwell G, Scheim A, Mitra S, Kerr T. Assessing support for supervised injection services among community stakeholders in London, Canada. Int J Drug Policy. 2017;48:2733. 